\title{
Computación en la nube: algunas consideraciones técnico-jurídicas*
}

\section{Computing in the cloud: Some Technical and Legal Considerations}

Gladys Stella Rodríguez (LUZ)** https://orcid.org/0000-0003-0063-1411

http://dx.doi.org/10.21503/lex.v17i23.1674

* Avance del proyecto de investigación registrado ante el Consejo de Desarrollo Científico y Humanístico (CONDES) adscrito al Vicerrectorado Académico de la Universidad del Zulia, intitulado: Principios básicos de la contratación en la nube.

** Abogada, magíster en Planificación y Gerencia de Ciencia y Tecnología. Doctora en Derecho. Postdoctora en Gerencia en las Organizaciones. Profesora de pregrado y postgrado en materia Informática Jurídica y Derecho Informático e Investigadora del Instituto de Filosofía del Derecho de la Facultad de Ciencias Jurídicas y Políticas de la Universidad del Zulia. Coordinadora del Programa de Postdoctorado en Derechos Humanos de LUZ. Investigadora acreditada en el Programa de Promoción para la Investigación e Innovación (PEII), Nivel C_Venezuela

Correo electrónico: gr1970ve@gmail.com

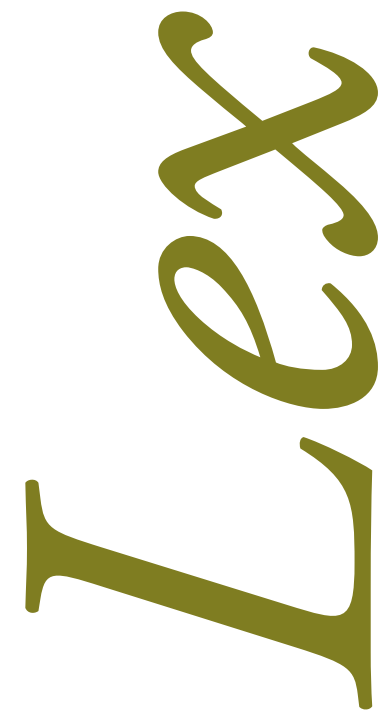




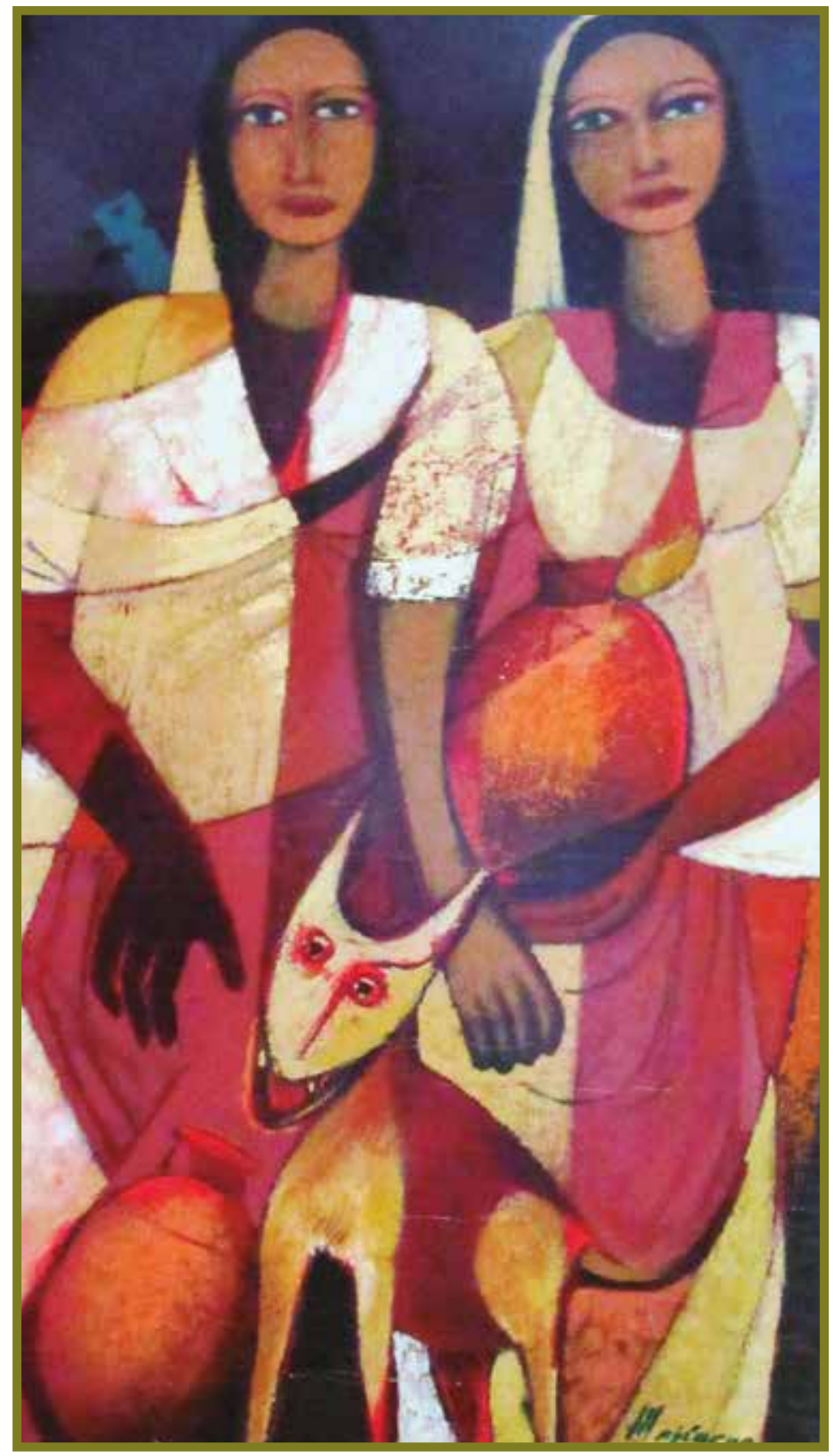

Mujeres con perro. Óleo 50 x $30 \mathrm{~cm}$.

Agustín Aquino Mejías (pintor peruano). 


\section{RESUMEN}

El presente artículo analiza algunas consideraciones técnico-jurídicas del contrato denominado computación en la nube, para ello, primeramente se expone su origen, se describe su naturaleza a partir de su definición siguiendo a Joyanes, García del Poyo y la Comisión de las Naciones Unidas para el Derecho Mercantil Internacional. De igual manera, se explican las características técnicas de este servicio tecnológico, se exponen los principales proveedores que ofrecen estos modelos de negocios, se examinan las ventajas y desventajas de este tipo de acuerdo y, finalmente, se analiza el aspecto contractual, con especial referencia a la fase preliminar, que tanto cliente como proveedor debe tomar en consideración a la hora de negociar y cumplir con el complejo de acuerdos que implica la contratación en la nube. Se desarrolla una investigación no experimental de carácter descriptivo, basada en el análisis de contenidos emanados por autores y organismos especializados en TIC, a la vez que se hace una interpretación de los documentos de la CNUDMI sobre los aspectos jurídicos.

Palabras clave: computación en la nube, modelos de negocios, aspecto contractual, fase preliminar.

\section{ABSTRACT}

This article describes some technical and legal considerations of the contract called Cloud Computing, for which purpose it starts by exposing its origin, its nature is described from its definition following (Joyanes, 2012), (García del Poyo, 2012) and the United Nations Commission for International Trade Law (UNCITRAL, 2017). Also, the technical features of this technological service are explained, its principal suppliers offering these business models are presented, and the advantages and disadvantages of this kind of agreement and finally the contractual aspect is analyzed with special reference to the preliminary phase which both the client and supplier should take into account when negotiating and complying with the series of agreements involved on cloud computing. A non-experimental descriptive investigation is developed based on analysis of the contents from authors and organisms specialized on ICT and at the same time, the UNCITRAL documents are interpreted on its legal aspects.

Key words: cloud computing; business models; contractual aspect; preliminary phase. 


\section{INTRODUCCIÓN}

En los últimos tiempos, los procesos relativos a la internacionalización, pero también a la deslocalización de las grandes empresas, en muchos casos transnacionales, han determinado que las necesidades de cómputo de las mismas hayan crecido de forma exponencial, si se las compara a cómo lo hacía la eventual capacidad de cálculo potencialmente inherente a los computadores personales de los que la misma disponía. ${ }^{1}$

Según el Boletín e-Gobierno de la Red Gobierno Electrónico de América Latina y el Caribe $^{2}$ las organizaciones privadas y estatales manifiestan una creciente tendencia a delegar en proveedores externos especializados el suministro (vía internet) de diversos recursos de Tecnologías de Información y Comunicación (TIC en adelante) — hardware, software, plataformas operativas, bases de datos, sistemas de seguridad- como servicios en línea, en vez de adquirirlos y mantenerlos por cuenta propia. Esta tercerización del suministro de las TIC se encuadra en el modelo tecnológico denominado cloud computing o "computación en la nube" (CN en adelante), en el cual los distintos recursos que integran una solución de TIC son provistos conjuntamente en el ciberespacio independientemente de su localización física, proporcionando al usuario un servicio confiable, continuado, elástico y asequible, por el que solo pagará por lo realmente consumido.

Uno de los temas más recurridos, en la actualidad, es, precisamente, el cloud computing o, en terminología castellana, "computación en la nube" — también denominada "nube TIC"— . Redes sociales como, entre otras, Facebook y MySpace, así como cuentas de correo electrónico basadas en la web, como el del popular Gmail del buscador Google, recurren a este servicio. Asimismo, como los hechos ponen de manifiesto, numerosos foros, blogs, noticias, conferencias de múltiples sectores o áreas y, en suma, diversos espacios, en vista de su interés, aluden a esta sugerente cuestión.

1 David López Jiménez, "La "computación en la nube" o "cloud computing” examinada desde el ordenamiento jurídico español”, Revista de Derecho de la Pontificia Universidad Católica de Valparaíso, XL (2013, 1er Semestre): 690.

2 Red de Gobierno Electrónico de América Latina y el Caribe (Red GEALC) Organización de los Estados Americanos (OEA), Banco Interamericano de Desarrollo (BID), Centro de Investigación para el Desarrollo Internacional (IDRC), "e-Gobierno en la nube", Boletín e-Gobierno Red GEALC 118 (octubre 2016): 3 
Otro aspecto que ha contribuido al desarrollo de esta categoría de relación jurídica-técnica, es el caso de la conexión a la red de redes, por ejemplo en el caso de Venezuela vale señalar que en el marco del evento Ganando con el Comercio Electrónico de la empresa Tendencias Digitales, realizado en 2018, expertos, entre ellos Ana Goite, ${ }^{3}$ destacan "que el 18,7 millones de personas se encuentran conectadas a Internet (..) lo cual calificó como un porcentaje bastante alto en comparación con otros países de la región aunado a que en el 2017 se produce un repunte principalmente por el uso de plataformas locales en el país como tiendas en línea y redes sociales".

Ahora bien, son varias las interrogantes que surgen en este tema: ¡en qué consiste este modelo de negocios tecnológicos en el ciberespacio?, ¿`uáles son los servicios que se ofrecen desde la nube?, ¿qué características resaltan en este tipo de acuerdo?, ¿cuáles plataformas digitales ofrecen esta modalidad de contrato, destacando las ventajas y riesgos que significa? Y, finalmente ¿se debe hacer un análisis de los lineamientos de los organismos internacionales a tomar en cuenta en materia de $\mathrm{CN}$, especialmente en su fase precontractual? Todo ello con el fin de analizar cuáles son los aspectos principales tanto técnicos como jurídicos de este novedoso contrato en la nube o red de redes, en el marco del organismo especializado de las Naciones Unidas.

De manera que en el presente trabajo se expondrán los orígenes de esta categoría de acuerdo que utiliza las TIC, se indicará la definición de este tipo de acuerdo siguiendo a algunos autores, organismos especializados y aportando una definición. Asimismo, se explicarán sus características y modelos, a la vez que se expondrán las ventajas y riesgos que este tipo de contrato involucra. Finalmente se analizará la naturaleza de este tipo de acuerdo en la nube en su fase precontractual, en el marco de los organismos internacionales competentes.

\section{ORÍGENES DEL CLOUD COMPUTING}

Como es sabido, el fenómeno de la computación en la nube ha recorrido, qué duda cabe, un largo camino desde que, por parte de ciertos investigadores, fue considerado una cuestión de una importante proyección futura. Su origen efectivo — sin perjuicio de las consideraciones que se realizarán - podría situarse a finales de la década de los noventa del siglo XX. Asimismo, debe matizarse que el vocablo nube básicamente se emplea como una metáfora de internet, fundamentado, en gran medida, en el dibujo de nubes utilizado en tiempos pretéritos para simbolizar a la red telefónica, y, posteriormente, para representar a internet en el ámbito de los diagramas de red de computadoras como una suerte de abstracción de la infraestructura subyacente que constituye.

3 Ana Goite, citada por Ricardo Zapata, “13,8 millones de venezolanos compraron o vieron una tienda online en 2017”, El Nacional Economía Empresas, 31 de mayo de 2018, acceso el 21 de enero de 2019, http://www.el-nacional.com/noticias/ empresas/138-millones-venezolanos-compraron-vieron-una-tienda-online-2017_237981 
A comienzos de la década de los sesenta, en concreto en 1962, J.C.R. Licklider de la empresa Bolt, Beranek and Newman (BBN), formuló un concepto de una red de computadoras susceptible de poder llegar a comunicar usuarios en diversos ordenadores personales. Douglas Parkhill, más de tres décadas después —en 1996—, se refirió a muchos de los caracteres inherentes a la computación en la nube. ${ }^{4}$ No obstante, a juicio de otros investigadores, ${ }^{5}$ los verdaderos orígenes de la computación en la nube se sitúan en la década de los cincuenta del siglo XX con Herb Grosch. Realmente, fue, precisamente, a finales de la década de los noventa, 1999, cuando Salesforce.com introdujo el concepto de entrega de aplicaciones empresariales a través de un sencillísimo sitio web. Posteriormente, solo tres años después, la popular empresa Amazon puso en práctica Amazon Web Service. Cuatro años más tarde, o sea, en 2006, llegó Google Docs ${ }^{6}$ que, como es conocido, popularizó el cloud computing. Naturalmente, además de los ejemplos enunciados, existen otros muchos, como Dropbox, Google Drive, iCloud de Apple y Onedrive de Microsoft.

Se trata de una modalidad de servicio de múltiples y complejas opciones transfronterizas o no; servicios susceptibles de clasificarse en infraestructuras (Infrastructure as a Service), plataformas de desarrollo (Platform as a Service), aplicaciones (Software as a Service) y finalmente procesos de negocio (Process as a Service). Como se verá, el servicio de computación en la nube supone la entrada de un tercero que no es sino el proveedor de servicios en la nube. Gran parte de la legislación aplicable a la materia que se comenta inicialmente no se elaboró, en modo alguno, pensando en la computación en la nube. ${ }^{7}$

\section{DEFINICIÓN DE CLOUD COMPUTING}

No solo es una frase de moda, es un término que representa un nuevo modelo de informática, tenido por muchos analistas como una innovación tan relevante como lo fue internet en su momento; además, es el mejor sinónimo de la propia web. Cloud computing es la evolución de un conjunto de tecnologías que afectan al enfoque de las organizaciones y empresas en la construcción de sus infraestructuras de TIC.

Al igual que ha sucedido con la evolución de la web, con la web 2.0 y la web semántica, la computación en la nube no incorpora nuevas tecnologías. Se han unido tecnologías potentes

4 M. García Sánchez, "Retos de la computación en nube", en Derecho y cloud computing, ed. por R. Martínez Martínez (Cizur Menor, Navarra: Thomson Reuters - Civitas, 2012), 37, determina que Parkhill fue el primer evangelista de este nuevo paradigma, en cuya esencia prima la idea de que la capacidad de computación debería de ser un servicio de acceso público universal como el agua, el gas o las telecomunicaciones.

5 David, López Jiménez, "La computación en la nube o cloud computing examinada desde el ordenamiento jurídico español”..., 693

6 El servicio de "Google Documentos y Hojas de cálculo", también denominado Google DocserSpreadsheets, constituye un programa gratuito basado en web para crear documentos en línea con la posibilidad de colaborar en grupo. Ahora bien, debemos poner de relieve que Google Docs recientemente ha sido reemplazado por Google Drive. Cada usuario tiene, de manera gratuita, cinco gigabytes de memoria con la finalidad de almacenar sus archivos.

7 Pues no es simple retórica, que el derecho suele ir por detrás de la realidad, lo que puede vislumbrarse en el ámbito tecnológico con mayor nitidez. 
e innovadoras, para construir este nuevo modelo y arquitectura de la web. Se plantea que, "si bien Internet es un fundamento necesario, la nube es algo más que Internet. Es aquel lugar donde utilizar tecnología cuando se necesita, mientras se necesite, ni un minuto más" ${ }^{8}$ No se instala nada en su escritorio, ni se paga por la tecnología cuando no se utiliza. La nube puede ser infraestructura o software, es decir, puede ser una aplicación a la que se accede a través del escritorio y se ejecuta inmediatamente tras su descarga, o bien un servidor al que se invoca cuando se necesita.

En la práctica, la informática en nube proporciona un servicio de software o hardware. No existe una definición aceptada universalmente; sin embargo, existen organismos internacionales cuyos objetivos son la estandarización de las TIC y, en particular, de cloud computing. Uno de los organismos más reconocidos es el National Institute of Standards and Technology $\left(\right.$ NIST) ${ }^{9}$ y su Information Technology Laboratory, que define la computación en nube (cloud computing) como:

Un modelo que permite el acceso bajo demanda a través de la red a un conjunto compartido de recursos de computación configurables (redes, servidores, almacenamiento, aplicaciones y servicios) que se pueden aprovisionar rápidamente con el mínimo esfuerzo de gestión o interacción del proveedor del servicio. ${ }^{10}$

Por su parte, afirma Luis Joyanes que "la nube es un conjunto de hardware y software, almacenamiento, servicios e interfaces que facilitan la entrada de la información como un servicio". ${ }^{11}$

El mismo autor considera igualmente que el mundo de la nube tiene un gran número de actores o participantes que son los siguientes: los vendedores o proveedores, quienes proporcionan las aplicaciones y facilitan las tecnologías, infraestructura, plataformas y la información correspondiente; los socios de los proveedores, quienes crean servicios para la nube, ofreciendo servicios a los clientes; los líderes de negocios, quienes evalúan los servicios de la nube para implantarlos en sus organizaciones y empresas; los usuarios finales, quienes utilizan los servicios de la nube, gratuitamente o con una tarifa ${ }^{12}$.

8 Luis Joyanes, “Computación en la nube. Notas para una estrategia española en Cloud Computing”, Revista del Instituto Español de Estudios Estratégicos, n 0 (2012): 92.

9 El NIST es una Agencia del Departamento de Comercio de los Estados Unidos. Dentro del NIST, el Computer Security Resource Center (CSRC) se encarga de los estándares de las Tecnologías de la Información y, en concreto, de cloud computing.

10 En octubre de 2009, Peter Mell y Tim Grance, investigadores del NIST, publicaron la norma (draft) de la definición de cloud computing y una guía del mismo realizada en colaboración con la industria y el gobierno, titulada: "Effectively and Securely Using the Cloud Computing Paradigm” y que puede ser descargada en el sitio oficial del NIST: http://crsc.nist. gov/groups/SN/cloud- computing/cloud-computing-v25.ppt

11 Luis Joyanes, "Computación en la nube. Notas para una estrategia espańola en Cloud Computing”..., 93

12 Luis Joyanes, "Computación en la nube. Notas para una estrategia espańola en Cloud Computing"..., 93 
Los servicios de la nube deben ser distribuidos (multi-tenancy); es decir, empresas diferentes comparten los mismos recursos fundamentales. Por esta razón, las empresas comienzan a encontrar nuevos valores, facilitando la eliminación de las complejas restricciones que supone el entorno informático tradicional; incluyendo espacio, tiempo, energía y costos.

García del Poyo conceptualiza esta categoría de la siguiente manera:

El cloud computing es un nuevo modelo de negocios a través del cual, mediante la utilización de tecnologías de la información y de las comunicaciones, se posibilita a los usuarios la capacidad de acceder a un catálogo de servicios estandarizados que sirven para responder a las necesidades de los individuos y de las empresas de una forma flexible y adaptativa. Desde el punto de vista tecnológico, el cloud computing puede definirse como un "paradigma de programación" que permite ofrecer servicios informáticos a través de internet. El término cloud o nube es obviamente una alusión metafórica a internet. ${ }^{13}$

Asimismo, la Secretaría de la Comisión de las Naciones Unidas para el Derecho Mercantil Internacional (CNUDMI en adelante) ofrece una definición en el Anexo "Modelo de capítulos de un posible texto de orientación sobre los aspectos contractuales de la computación en la nube", correspondiente al papel de trabajo intitulado: "Aspectos contractuales de la computación en la nube", el cual señala:

2. La computación en la nube puede definirse, en términos generales, como el suministro y el uso de servicios de computación (por ejemplo, hospedaje de datos o procesamiento de datos) a través de redes abiertas o cerradas. Los contratos de servicios de nube son, por lo tanto, una clase especial de contratos de prestación de servicios. Según cuáles sean los datos objeto de las actividades de computación en la nube, los contratos de servicios de nube pueden estar sujetos a diversos regímenes jurídicos, entre ellos las leyes de protección de la privacidad, el derecho bancario y la normativa contra el blanqueo de dinero. Este tipo de contratos suele tener una dimensión internacional o transfronteriza, pero la legislación o la práctica pueden también circunscribir la computación en la nube a una sola jurisdicción. ${ }^{14}$

De lo anterior se puede seńalar que se trata de un modelo complejo de contratos de servicios de negocios tecnológicos, que involucra acuerdos precontractuales y cuyo objeto puede ir desde implementar soluciones de TIC más dinámicas y ágiles, capaz de crear productos y servicios en la nube o web; modelar nuevos productos o campañas de marketing como pro-

13 Rafael García del Poyo, “Cloud Computing: Aspectos jurídicos clave para la contratación de estos servicios”, Revista Española de Relaciones Internacionales, n. ${ }^{\circ} 2$ (2012): 48-49, acceso el 29 de abril de 2017, http://reri.difusionjuridica.es/index. $\mathrm{php} / \mathrm{RERI} /$ article/view/45/43

14 Asamblea General de las Naciones Unidas, Comisión de las Naciones Unidas para el Derecho Mercantil Internacional, Grupo de Trabajo IV (Comercio Electrónico), 55 periodo de sesiones, "Aspectos contractuales de la computación en la nube”, Documento A/CN.9/WG.IV/WP.142, Nueva York, 24 a 28 de abril de 2017; 13. 
totipos de software basados en ese ambiente de red de redes; ofrecer un depósito común para la recopilación y análisis de nuevos datos, hasta configurar un espacio donde se realizan un número creciente de operaciones de inteligencia artificial, como reconocimiento de imagen y voz.

Otra forma de entender esta categoría técnico-jurídica en ascenso es visualizarla como redes computacionales que actúan como un disco duro con enormes centros de procesamiento de datos, lo cual implica una pluralidad de actores intervinientes, capaces de guardar imágenes, documentos y datos de sitios web, a cargo de una contraprestación para crearlas y ofrecer un servicio rápido, significando una serie de características que se enlazan y es preciso comprender.

\section{CARACTERÍSTICAS DE CLOUD COMPUTING}

El modelo de la nube, según NIST, se compone de cinco características esenciales:

o Autoservicio bajo demanda o Acceso personalizado. Un consumidor puede proveerse unilateralmente de tiempo de servidor y almacenamiento en red, a medida que lo necesite, sin requerir interacción humana con el proveedor del servicio. Como afirma García del Poyo, ${ }^{15}$ basta con que tenga una adecuada infraestructura de telecomunicaciones que le facilite acceso a internet, no se precisa instalación de ningún software adicional, ya que - habitualmente- suelen usarse browsers o buscadores para acceder a todos los servicios ofrecidos en la nube.

o Acceso ubicuo a la red. Se realiza mediante mecanismos estándares, que promueven el uso por plataformas de clientes delgados (teléfonos móviles, computadoras portátiles, PDA, ${ }^{16}$ tabletas).

o Distribución de recursos independientes de la posición o multiposesión. Los recursos de computación del proveedor son agrupados (pooled) para servir a múltiples consumidores utilizando un modelo multi-distribuido (multitenant) con diferentes recursos físicos y virtuales asignados y reasignados dinámicamente conforme a la demanda del consumidor.

Según García del Poyo, ${ }^{17}$ se basa en información que es almacenada de manera permanente en servidores o data centers, accesibles a través de internet, pero esta comunicación con

15 Rafael García del Poyo, “Cloud Computing: Aspectos jurídicos clave para la contratación de estos servicios”..., 49.

16 PDA o Personal Digital Assistant (en español, Asistente Personal Digital), son un tipo de handheld (computadora de mano). Pequeńas computadoras que entran en la mano que tienen un sistema y programas con tecnologías de inteligencia artificial y que ayudan sus usuarios en ciertas actividades como la búsqueda de información, agenda electrónica, etc. Poseen reconocimiento de escritura.

17 Rafael García del Poyo, "Cloud Computing: Aspectos jurídicos clave para la contratación de estos servicios"..., 49. 
los servidores no se realiza de forma única por un solo cliente, de modo que los mismos son compartidos por multitud de usuarios.

Es evidente que se produce una sensación de independencia de la posición, de modo que el cliente, normalmente, no tiene control ni conocimiento sobre la posición exacta de los recursos proporcionados. Pero podría especificarla a un nivel más alto de abstracción (país, región geográfica o centro de datos). Ejemplos de recursos incluyen almacenamiento, procesamiento, memoria, ancho de banda de la red y máquinas virtuales.

o Elasticidad rápida. Las funcionalidades (capabilities) se pueden proporcionar de modo rápido y elástico, en algunos casos automáticamente. Sus características de aprovisionamiento dan la sensación de ser ilimitadas y pueden adquirirse en cualquier cantidad o momento.

o Servicio medido. Los sistemas de computación en la nube controlan y optimizan automáticamente el uso de recursos, potenciando la capacidad de medición en un nivel de abstracción apropiado al tipo de servicio (almacenamiento, procesamiento, ancho de banda y cuentas activas de usuario). El uso de recursos puede ser monitorizado, controlado e informado, proporcionando transparencia para el proveedor y para el consumidor. El uso de estos recursos por parte de los clientes puede seguirse, controlarse y notificarse, lo que aporta una enorme transparencia en la gestión del negocio para ambas partes.

\section{MODELOS DE LA NUBE}

Siguiendo el NIST, los modelos de la computación en nube se clasifican en dos grandes categorías:

A) Modelos de despliegue. Se refieren a la posición (localización) y administración (gestión) de la infraestructura de la nube. Son cuatro modelos: pública, privada, híbrida y comunitaria.

A.1) Nube privada. Los servicios no son ofrecidos al público en general. La infraestructura es íntegramente gestionada por una organización. La nube privada es un servicio basado en recursos propios de una empresa, o prestado a una organización en exclusiva, no compartido con otras organizaciones, pero que se paga bajo el modelo por demanda.

A.2) Nube pública. La infraestructura es operada por un proveedor que ofrece servicios al público en general. Se conoce como nube pública a los servicios compartidos que ofrecen los grandes proveedores, como por ejemplo los 
Amazon Web Services para empresas, o el correo electrónico de Microsoft o de Google. ${ }^{18}$

A.3) Nube híbrida. Resultado de la combinación de dos o más nubes individuales que pueden ser privadas, compartidas o públicas. Permite enviar datos o aplicaciones entre ellas.

A.4) Nube comunitaria (community). Ha sido organizada para servir a una función o propósito común. Es preciso compartir objetivos comunes (misión, políticas, seguridad). Puede ser administrada bien por las organizaciones constituyentes, bien por terceras partes. Este modelo es el definido por el NIST, aunque la mayoría de organizaciones, proveedores y usuarios de la nube aceptan los tres modelos de despliegue: pública, privada e híbrida.

B) Modelos de servicio. Se refieren a los servicios específicos a los que se puede acceder en una plataforma de $\mathrm{CN}$, son tres los modelos: software, plataforma e infraestructura como servicios.

B.1.) Servicios de software. Al usuario se le ofrece la capacidad de que las aplicaciones suministradas se desenvuelvan en una infraestructura de la nube, siendo las aplicaciones accesibles a través de un navegador web, como en el correo electrónico web. Posiblemente, este es el ejemplo más representativo, por lo extendido, de este modelo de servicio. El usuario carece de cualquier control sobre la infraestructura o sobre las propias aplicaciones, excepción hecha de las posibles configuraciones de usuario o personalizaciones que se le permitan. Se alquila el software que funciona en los servidores y se paga una renta mensual en lugar de comprar licencias.

B.2) Servicios de plataforma. Al usuario se le permite desplegar aplicaciones propias (adquiridas o desarrolladas por el propio usuario) en la infraestructura de la nube de su proveedor, que ofrece la plataforma de desarrollo y las herramientas de programación. En este caso, el usuario mantiene el control de la aplicación, aunque no de toda la infraestructura subyacente. Se ofrece a desarrolladores de software para crear aplicaciones y venderlas en la misma plataforma.

18 Anónimo, "La computación en la nube cambia el paradigma de los negocios", Dinero (26 de mayo de 2016), acceso el 17 de enero de 2019, https://www.dinero.com/edicion-impresa/tecnologia/articulo/la-computacion-en-la-nube-cambia-el-paradigma-de-los-negocios/224009 
B.3) Servicios de infraestructura. El proveedor ofrece recursos como capacidad de procesamiento, de almacenamiento o comunicaciones, que el usuario puede utilizar para ejecutar cualquier software; desde sistemas operativos hasta aplicaciones. Se alquila espacio en servidores para almacenar información y se alquila capacidad de cómputo en servidores. Incluye bases de datos, conectividad, hosting, ${ }^{19}$ streaming, ${ }^{20}$ entre otros servicios.

\section{ALGUNOS PROVEEDORES DE NEGOCIOS EN LA NUBE}

Está totalmente enfocado hacia las características clave de la computación en la nube, realizado para potenciar sus conceptos (tecnologías y modelos de ingresos). Los modelos de negocios se pueden aplicar por igual a proveedores y consumidores de la nube. El de proveedores se basa en el desarrollo de tecnologías y soluciones facilitadoras de la nube; incluye las siguientes soluciones: ${ }^{21}$

- Proveedores de servicios de la nube. Proporcionan la red e infraestructuras de computación mediante plataformas y soluciones. Los proveedores de servicios de la nube incluyen organizaciones que operan con centros de datos propios y apoyados en servicios de virtualización. Los proveedores son variados y tienen gran implantación, aprovechando sus centros de datos y de su experiencia en alojamiento de datos y aplicaciones.

Proporcionan plataformas basadas en la nube, hospedados en entornos de sistemas e infraestructuras específicos, para que los desarrolladores puedan acceder a la plataforma, desarrollar una nueva aplicación de negocios y alojarlas en la plataforma basada en la nube.

Esta incluye servidores, redes y almacenamiento de datos y es la de mayor adopción. De lejos, el jugador más grande en este segmento es Amazon Web Services (AWS), que junto con Microsoft y su servicio Azure, son las dos compañías globales que lideran el llamado "cuadrante mágico de Gartner", un ranking que esta consultora realiza con base en estudios de mercado mundiales. Pero Amazon Web Services ofrece más capacidad de cómputo que todas las otras 14 compañías juntas que figuran en el ranking de Gartner, en donde se destacan también VMware, Google e IBM. El éxito de Amazon radica en que "no deja de innovar y sus precios son muy atractivos", afirma Diego Maldonado, presidente de Avanxo, uno de los socios de

19 Servicio que ofrecen algunas compañías (los webhost) en Internet que consiste en ceder un espacio en sus servidores para subir (alojar, hostear) un sitio web para que pueda ser accedido en todo momento en forma on line (acceso el 21-01-19, www.alegsa.com.ar).

20 Término que hace referencia al hecho de transmitir video o audio remotamente a través de una red (como internet) en tiempo real sin necesidad de descargar el archivo completo. Por ejemplo cuando se trasmite una radio o un canal de televisión en vivo por internet (acceso el 21-01-19, www.alegsa.com.ar).

21 Eric A. Marks y Bob Lozano, Executive's Guide to Cloud Computing (New Jersey: Wiley, 2010), 82-83. 
negocio más grande de $A W S$ y de Salesforce en Latinoamérica ${ }^{22}$

- Proveedores de tecnologías. Desarrollan las herramientas y tecnologías que facilitan que la nube se establezca y proporcione a los consumidores recursos proporcionados por la nube. Ofrecen un amplio rango de herramientas, tecnologías, sistemas operativos para facilitar el despliegue de nubes públicas, privadas, híbridas y comunitarias.

El líder mundial es Salesforce, cuyas soluciones para la gestión de negocios son conocidas, aunque los proveedores tradicionales de software y plataformas para la gestión de los negocios, como $\mathrm{SAP}^{23}$ y Oracle, ${ }^{24}$ también tienen sus plataformas bajo el modelo cloud. "Los clientes se han dado cuenta de que sus esquemas de operación anteriores eran demasiado costosos e ineficientes. Buscan soluciones más sencillas y eficientes”, explica Carlos Hernández, ${ }^{25}$ responsable de servicios financieros para el norte de Latinoamérica de SAP.

- Proveedores de soluciones. Estos están más cercanos al consumidor final. Para atraer a sus primeros clientes, Dropbox hizo videos promocionales que generaron discusión en sitios web como Reddit y Slashdot. La estrategia funcionó: más de 5000 usuarios estaban en la lista de espera y en pocos días Dropbox logró más de 75000 suscriptores. Después, pasó de 100 000 usuarios a 200000 en unos 10 días. Hoy Dropbox tiene más de 500 millones de usuarios registrados, 11,5 millones de los cuales pagan una suscripción anual para tener más espacio de almacenamiento que el que ofrece la versión gratuita. ${ }^{26}$

- Modelos de negocio para consumidores. Otra aplicación cercana a consumidores, pero ya de negocios, son empresas que ofrecen aplicaciones de conceptos de la nube a las distintas estrategias de negocios. Ofrecen soluciones para gestión empresarial.

22 Anónimo, "La computación en la nube cambia el paradigma de los negocios", Dinero (26 de mayo de 2016), acceso el 17 de enero de 2019, https://www.dinero.com/edicion-impresa/tecnologia/articulo/la-computacion-en-la-nube-cambia-el-paradigma-de-los-negocios/224009

23 SAP fue fundada en junio de 1969 como Systemanalyse und Programmentwicklung ("Análisis de Sistemas y Desarrollo de Programas") por cinco ex ingenieros de IBM en Mannheim, Baden-Württemberg (DietmarHopp, Klaus Tschira, Hans-Werner Hector, Hasso Plattner, y Claus Wellenreuther). El acrónimo fue cambiado más adelante para ser Systeme, Anwendungenund Produkte in der Datenverarbeitung ("Sistemas, Aplicaciones y Productos en Procesamiento de Datos").

24 Oracle Corporation es una compañía especializada en el desarrollo de soluciones de nube y locales. Oracle tiene su sede en la localidad californiana. Es la primera compañía de software de estereotipos que desarrolla e implementa aplicaciones para empresas, cien por ciento activada por Internet a través de toda su línea de productos: base de datos, aplicaciones comerciales y herramientas de desarrollo de aplicaciones y soporte de decisiones. Oracle es el proveedor mundial de software para administración de información a lo largo de todo el mundo,

25 Anónimo, "La computación en la nube cambia el paradigma de los negocios", Dinero, 26 de mayo de 2016, acceso el 17 de enero de 2019, https://www.dinero.com/edicion-impresa/tecnologia/articulo/la-computacion-en-la-nube-cambia-el-paradigma-de-los-negocios/224009

26 Will Smale, "Cómo Drew Houston y Arash Ferdows triunfaron con Dropbox, una empresa tecnológica que Steve Jobs quiso destruir”, BBC News. Economía (17 de julio de 2018), acceso 21 de enero de 2019, https://www.bbc.com/mundo/ noticias- 44847080 


\section{VENTAJAS DEL CLOUD COMPUTING}

El servicio de computación en la nube tiene un conjunto de ventajas de múltiples tipos. Así, entre otros, las de carácter tecnológico, ambiental, pero también social en diversos colectivos. Por lo que respecta a las empresas que recurren a los servicios susceptibles de ser incardinados en la computación en la nube se pueden, entre otras, mencionar las siguientes prerrogativas: de tipo económico-financiero - ahorro de costos de capital, control de costos y beneficios de tipo marginal—, foco en el negocio, continuidad de negocio y capacidad de recuperación frente a eventuales desastres, incremento de los recursos disponibles, modernización de los procesos de negocio, celeridad, escalabilidad y flexibilidad, seguridad, diversificación de los sistemas de TIC, evaluación de viabilidad y rentabilidad de posibles nuevos servicios, movilidad y plena disponibilidad.

En este último sentido, no se necesita espacio físico alguno para poder almacenar servidores y bases de datos, ya que, como es conocido, están, como su propio nombre lo indica, en la nube. Sin perjuicio de las ventajas comentadas, cabe también señalar otras muchas como el incremento de la productividad de las empresas, la sensible mejora de los servicios públicos, así como de la calidad de vida y, finalmente, la evolución más avanzada hacia ciertos modelos de TIC.

Ahora bien, el servicio $\mathrm{CN}$ también presenta múltiples ventajas para los ciudadanos y para las propias Administraciones de carácter público. Por lo que se refiere a estas últimas, el modelo cloud puede facilitar la generalización de los servicios transversales a toda la Administración, con la correspondiente mejora de la eficiencia y la mayor reutilización de la infraestructura tecnológica de las Administraciones Públicas. Así, en el ámbito local, que, dicho sea de paso, está sensiblemente menos dotado de recursos técnicos y humanos, puede desplegar una loable y necesaria labor para la modernización de sus procesos. En cuanto a los ciudadanos, la tecnología que comentamos, en clara convergencia con la introducción de las redes sociales y el desarrollo de la tecnología de virtualización, ha tenido un notable impacto en la sociedad.

\section{RIESGOS Y AMENAZAS EN CLOUD COMPUTING}

La publicación del NIST (National Institute of Standards and Technologies) "Guide lines on Security and Privacy in Public Cloud Computing" pone de manifiesto, además de la actualidad de este nuevo modelo para la distribución de servicios y aplicaciones, la necesidad de difundir buenas prácticas de seguridad. Este no es el único documento que refleja la creciente preocupación por la seguridad en estas plataformas, como se refleja en informes de otras entidades de referencia. El informe "Riesgos y amenazas en el Cloud Computing" realizado 
por INTECO ${ }^{27}$ resume algunos de estos documentos con el propósito de facilitar una visión general de amenazas, riesgos y aspectos claves en la seguridad en cloud.

Este informe describe las infraestructuras y servicios cloud, analizando los distintos elementos que han de tenerse en cuenta para su seguridad, según los criterios y estándares internacionales. Las preocupaciones que derivan de estos informes se centran en la gestión de los datos, fundamentalmente en su propiedad y forma de operarlos y tratarlos. Con el análisis realizado en este informe, se obtiene una visión global de esta problemática y se extraen conclusiones comunes a todos los puntos de vista. La seguridad y la propiedad de los datos es uno de los aspectos clave. Los informes muestran una gran preocupación por la propiedad y el tratamiento de los datos, dado que estas infraestructuras pueden gestionarlos en múltiples países, lo que puede generar conflictos en cuanto al marco legal en el que son tratados. También se plantea que estos entornos, al manejar gran cantidad de datos, pueden ser objeto de fugas de información, ya sean intencionadas o fortuitas.

El cumplimiento normativo también es uno de los pilares de la seguridad en entornos cloud. En este caso, el problema se presenta debido a la falta de transparencia de estas infraestructuras, por lo que es recomendable que el suscriptor del servicio se informe claramente de cómo se gestiona el entorno. Para la creación de un servicio cloud, interviene una multitud de softwares de distintos proveedores. Al ser entornos complejos, hay que vigilar las posibles vulnerabilidades del mismo e implantar procedimientos de parcheado.

Otro de los aspectos considerados importantes es la identidad y el control de acceso. Por lo general, la mayoría de las infraestructuras son compartidas por múltiples empresas o usuarios y su mala definición puede provocar accesos no autorizados a datos confidenciales. La definición de una buena política de identidad y control de acceso, basada en el mínimo privilegio, es esencial en entornos cloud. Por último, los contratos de acuerdo de servicio (SLA) son aquí un denominador común. Todas las recomendaciones indican que deben ser revisados y creados específicamente, detallando los controles, normativas, medidas de protección, plazos de recuperación del servicio. ENISA, la agencia de seguridad de la Unión Europea, ha publicado recientemente una normativa específica para contratos en la nube. Y organismos como la CNUDMl, también han venido trabajando en algunos lineamientos, lo cual se examinará a continuación, por ser de carácter más universal.

27 INTECO. Este informe de INTECO se preparó con base en la publicación de ENISA (Agencia Europea de Seguridad), "Seguridad y resistencia en las nubes de la Administración Pública", ENISA, acceso el 17 de enero de 2019, www.enisa. europa.eu/ 


\section{ALGUNAS CONSIDERACIONES JURÍDICAS DE LA CONTRATACIÓN EN LA NUBE}

A fines de hacerse el análisis correspondiente, se acude a varios documentos de la CNUDMI, Grupo de Trabajo IV (Comercio Electrónico). Se inicia el análisis con el documento emanado del mandato otorgado en el $55^{\circ}$ período de sesiones. ${ }^{28}$ En esta ocasión, se sugirieron algunas medidas que podría adoptar la Comisión con respecto a una propuesta presentada por Canadá, titulada "Posible labor futura en materia de comercio electrónico: cuestiones jurídicas que afectan a la computación en la nube" (A/CN.9/823 y A/CN.9/856). En esta sesión $55^{\circ}$, además de examinar informes, normas y publicaciones relacionados con el tema, la Secretaría mantuvo consultas oficiosas con expertos. Se trató de que la participación de expertos en las consultas oficiosas fuese lo más amplia posible para que estuvieran representados los puntos de vista de todas las regiones, de los principales sistemas económicos y jurídicos del mundo y de los países desarrollados y en desarrollo.

En primer lugar, la Secretaría pidió a los expertos que opinaran sobre la lista de cuestiones que se proponía que se abordaran en un texto que prepararían la CNUDMI o su secretaría sobre el tema de la computación en la nube. Los comentarios recibidos sirvieron de base para definir la estructura y el contenido del texto que finalmente se envió a los expertos en forma de proyecto de guía jurídica sobre los aspectos contractuales de la computación en la nube, para que formularan observaciones al respecto. El proyecto de guía jurídica suscitó numerosas observaciones:

- Existe la preferencia por un texto no legislativo en el que se analizarán cuestiones contractuales relacionadas con la computación en la nube y los posibles criterios aplicables a esas cuestiones, ello debido a que la computación en la nube planteaba cuestiones delicadas en materia de políticas, como la protección de los datos personales y aspectos jurisdiccionales.

- Se cuestionó si los aspectos jurídicos que planteaban las relaciones contractuales en el ámbito de la computación en la nube eran tan diferentes de los vinculados a otros tipos de contratos, como por ejemplo la contratación externa de servicios informáticos, o los contratos de arrendamiento, prestación de servicios o concesión de licencias de tecnología de la información, como para justificar que se preparara una guía jurídica detallada sobre la computación en la nube, similar a las guías jurídicas ya existentes de la CNUDMI.

28 Asamblea General de las Naciones Unidas, Comisión de las Naciones Unidas para el Derecho Mercantil Internacional, Grupo de Trabajo IV (Comercio Electrónico), 55 periodo de sesiones, "Aspectos contractuales de la computación en la nube", Documento A/CN.9/WG.IV/WP.142, Nueva York, 24 a 28 de abril de 2017; 20. 
- Existe preocupación de que una guía jurídica detallada pudiera volverse obsoleta en poco tiempo debido a la rápida evolución de las prácticas contractuales en el sector de la computación en la nube.

- Cabe la posibilidad de que en algunas jurisdicciones la $\mathrm{CN}$ estuviera sometida a los principios aplicables a las empresas de servicios públicos (por ejemplo, el suministro de servicios seguros y adecuados a todo aquel que lo solicite, sin discriminación indebida y a precios justos y razonables), lo que limitaría considerablemente la libertad contractual de los proveedores de servicios de nube.

En consecuencia, se sugirió que se preparara un texto de orientación breve, sobre el que sería más fácil ponerse de acuerdo y que resultaría más accesible para los usuarios. Efectivamente, de conformidad con la recomendación del Grupo de Trabajo sobre la posible labor futura en materia de computación en la nube (A/CN.9/902, párr. 23) y las opiniones expresadas al respecto en el $50^{\circ}$ período de sesiones de la Comisión, ${ }^{29}$ la Secretaría presenta al Grupo de Trabajo para su examen un proyecto de lista de verificación que incluye las principales cuestiones relacionadas con los contratos de computación en la nube. El proyecto de lista de verificación, elaborado por la Secretaría con la participación de expertos, refleja las consideraciones preliminares formuladas por el Grupo de Trabajo en lo que respecta al ámbito de aplicación y al contenido que podría tener una lista de verificación, así como a los criterios para su redacción (A/CN.9/902, párrs. 11 a 28).

\section{ASPECTOS PRECONTRACTUALES DE LA CONTRATACIÓN EN LA NUBE}

La denominada lista de verificación ${ }^{30}$ consta de dos partes y un glosario. En la primera parte se abordan los principales aspectos precontractuales que las futuras partes, primordialmente el cliente, podrían tener en cuenta antes de firmar un contrato de computación en la nube. En la segunda, se abordan las principales cuestiones contractuales que las partes podrían necesitar resolver al redactar un contrato de computación en la nube; y en el glosario se describen algunos de los términos técnicos utilizados en la lista a fin de facilitar su comprensión.

En este trabajo, a fin del análisis correspondiente, se tomará solo la fase precontractual. Se entiende que en algunas jurisdicciones, la información precontractual puede considerarse parte integrante de este tipo de contrato. La lista de verificación presenta tres aspectos precontractuales, a saber: a) verificación de la existencia de normas imperativas y otros requisitos; b) evaluación precontractual de riesgos y c) otros aspectos precontractuales, tales como reve-

29 Documentos Oficiales de la Asamblea General, septuagésimo segundo período de sesiones, Suplemento núm. 17 (A/72/17), párrs. 116 a 127.

30 Asamblea General de las Naciones Unidas, Comisión de las Naciones Unidas para el Derecho Mercantil Internacional, Grupo de Trabajo IV (Comercio Electrónico), 56 periodo de sesiones, "Aspectos contractuales de la computación en la nube", Documento A/CN.9/WG.IV/WP.148, Nueva York, 16 a 20 de abril de 2018; 5. 
lación de información y confidencialidad.

a) Verificación de la existencia de normas imperativas y otros requisitos. ${ }^{31}$ Este primer aspecto se explica por sí solo, debido a que el marco jurídico aplicable al cliente, al proveedor o a ambos puede imponer ciertas condiciones para la celebración de un contrato de computación en la nube y, con ello no se violenta el principio de autonomía de la voluntad, dado que se trata de normas imperativas que cada Estado establece y a las cuales se deben someter los acuerdos que se celebren, pues el incumplimiento de los requisitos obligatorios puede acarrear importantes consecuencias negativas como la invalidez o la inexigibilidad del contrato, o de una parte del mismo, multas administrativas y responsabilidad penal. Por ejemplo, pueden incluir la obligación de adoptar medidas especiales para proteger los derechos de los sujetos de los datos, desplegar un determinado modelo de servicio (nube privada, en lugar de pública), cifrar los datos alojados en la nube y registrar ante las autoridades del Estado una transacción o un programa informático utilizado en el tratamiento de los datos personales. También pueden incluir requisitos de ubicación de los datos, así como requisitos relativos al proveedor.

En este orden resulta de interés los dos últimos aspectos precontractuales, se hace referencia a cada uno:

b) Evaluación precontractual de riesgos. ${ }^{32}$ En la evaluación precontractual de riesgos, una evaluación de riesgos podría ayudar a determinar cuáles son las medidas más adecuadas para mitigar tales riesgos, como la negociación de ciertas cláusulas. Por lo cual, si el Estado no exige que se realice una evaluación de riesgos como condición para celebrar un contrato de computación en la nube, se recomienda llevar a cabo tal evaluación.

Por otra parte, algunos riesgos no son intrínsecos al contrato en la nube, sino que podrían tener que abordarse de forma independiente al contrato de computación en la nube (por ejemplo, los riesgos derivados de las interrupciones en la conexión a Internet), ${ }^{33}$ y no todos

31 Asamblea General de las Naciones Unidas, Comisión de las Naciones Unidas para el Derecho Mercantil Internacional, Grupo de Trabajo IV (Comercio Electrónico), 56 periodo de sesiones, "Aspectos contractuales de la computación en la nube”, Documento A/CN.9/WG.IV/WP.148, Nueva York, 16 a 20 de abril de 2018; 7.

32 Asamblea General de las Naciones Unidas, Comisión de las Naciones Unidas para el Derecho Mercantil Internacional, Grupo de Trabajo IV (Comercio Electrónico), 56 periodo de sesiones, "Aspectos contractuales de la computación en la nube", Documento A/CN.9/WG.IV/WP.148, Nueva York, 16 a 20 de abril de 2018; 8-10.

33 Según el informe "Estado de la banda ancha en América Latina y el Caribe 2016" de la CEPAL, se señala que en términos de calidad del servicio, "ningún país de la región tiene al menos $5 \%$ de sus conexiones con velocidades mayores de $15 \mathrm{Mbps}$ mientras que, en los países avanzados, el porcentaje de conexiones de esta velocidad es cercano al $50 \%$ \%. En Banda Ancha Fija, la media de la velocidad promedio era de 4,7 Mbps, con Chile como el país con mayor velocidad (7,3 Mbps) y Venezuela con la menor (1,9 Mbps). La velocidad de acceso de la Banda Ancha Fija es clave para los procesos productivos y el salto que debiera dar América Latina para aumentar sus índices de crecimiento económico, aspecto que hay que tomar en cuenta cuando se celebra este tipo de acuerdo en países de la Región, acceso el 10 de septiembre de 2017, http://www.el-nacional.com/noticias/historico/cepal-venezuela-tiene-velocidad-internet-mas-lenta-latinoamerica_7514. Por su parte, Patricia Sanjorge señala que Chile vuelve a liderar este año el ranking mundial de competitividad 
pueden mitigarse a un costo aceptable (por ejemplo, los daños a la reputación, al honor). Además, se recomienda que la evaluación de riesgos se lleve a cabo continuamente antes y durante la vigencia del contrato y que de ello resulte la modificación o resolución de este.

Otro aspecto importante en materia de riesgo es verificar el historial del proveedor elegido, ello contribuirá a ponderar los posibles riesgos de contratar con uno u otro proveedor. Por ejemplo, cuáles son las políticas de privacidad, confidencialidad y seguridad de ese proveedor, en especial, en lo que respecta a la prevención de accesos no autorizados, la utilización, la alteración o la destrucción de los datos del cliente durante el tratamiento, el tránsito o la transmisión a los sistemas del proveedor y hacia fuera de ellos. Una situación delicada se ha presentado con icloud de Apple. ${ }^{34}$

Otro aspecto en torno a la elección del proveedor a considerar son las garantías ofrecidas al cliente de acceso permanente a los metadatos, registros de auditoría; la existencia de un plan de recuperación en casos de desastre y las obligaciones de notificación en caso de violación de la seguridad o mal funcionamiento del sistema; el hecho de que el proveedor ofrezca asistencia en los procesos de migración a la nube y finalización del servicio, así como garantías de interoperabilidad y transferibilidad; las medidas existentes de investigación de los antecedentes y capacitación de empleados, subcontratistas y otros terceros que participen en la prestación de los servicios de computación en la nube; las estadísticas de incidentes de seguridad e información sobre la calidad del servicio; la certificación otorgada por un tercero independiente que acredite el cumplimiento de las normas técnicas; la acreditación de la periodicidad y el alcance de la auditoría realizada por un órgano independiente; la solvencia financiera del proveedor; las pólizas de seguro contratadas por el proveedor; los posibles

digital en América Latina, situándose en el puesto trigésimo séptimo del nuevo informe publicado por el Centro Mundial de Competitividad de la escuela de negocios IMD, que este ańo lidera Estados Unidos, Chile, México y Argentina. El ranking mundial de IMD analiza sesenta y tres economías del mundo en el ámbito digital y el objetivo de la clasificación es evaluar la medida en que un país adopta y explora las tecnologías digitales que conducen a la transformación de las prácticas gubernamentales, los modelos comerciales y la sociedad en general. El ranking de IMD está basado en cincuenta indicadores que a su vez se engloban en tres grandes áreas: conocimiento, tecnología y preparación. Tanto Brasil como Perú, Venezuela y Colombia se sitúan en la parte inferior del índice elaborado por IMD, acceso el 19 de julio de 2018, http://www.el-nacional.com/noticias/latinoamerica/chile-mantiene-primer-puesto-competitividad-digital-latinoamerica_244498.

34 En 2017, Apple anunció que se asociaría con la firma local china, Guizhou-Cloud Big Data Industry Development [GCBD], para el manejo de las operaciones de los servicios de iCloud para los usuarios en China continental. En febrero de este año, se anunció que los datos de iCloud de los usuarios en China se trasladarían a un nuevo centro de datos en la provincia de Guizhou. Apple dijo que el cambio se hizo para que pueda cumplir con las leyes locales de seguridad cibernética. Ese acuerdo ahora se ha complicado por un nuevo acuerdo entre el operador $i$ Cloud chino de Apple y la empresa estatal, China Telecom. Apple confirmó a la BBC que la división Tiany iCloud de China Telecom firmó con GCBD para proporcionar almacenamiento para $i$ Cloud China. El cambio en la operación significa que los datos de los usuarios de $i$ Cloud en China, incluidos correos electrónicos y fotos, ahora son manejados por el servicio estatal. Esta medida que ha despertado la preocupación de los defensores de la privacidad, ansiosos de que esto le brinde al Gobierno chino un acceso más fácil a la información personal, acceso el 19 de julio de 2018, https://www.bbc.co.uk/news/technology-44870508 
conflictos de intereses; y el alcance de la subcontratación y de los servicios estratificados de computación en la nube.

Otro aspecto es que hay que asegurarse de antemano de que la utilización de los servicios de computación en la nube no supondrá una violación de derechos de propiedad intelectual ni una causa de revocación de la licencia concedida a cualquiera de las partes. Los costos de incurrir en una violación de derechos de propiedad intelectual pueden ser muy elevados. Es posible que sea necesario pactar el derecho para conceder sublicencias o celebrar un contrato directo de licencia con el correspondiente tercero licenciante que otorgue el derecho a gestionar las licencias que sean propiedad de terceros. Para utilizar programas informáticos de código abierto u otros contenidos, podría ser necesario obtener por anticipado el consentimiento de terceros y revelar el código fuente con las modificaciones introducidas en tales programas y otros contenidos. ${ }^{35}$

El cliente también debería considerar cuidadosamente el efecto que puede tener la duración del contrato. En los contratos a largo plazo y en aquellos a corto y medio plazo que se renuevan automáticamente puede existir un mayor riesgo de dependencia tecnológica, una estrategia importante para reducir los riesgos de dependencia puede ser la de contratar con varios proveedores y optar por una combinación de varios tipos de servicios de computación en la nube y sus modelos de despliegue (por ejemplo, emplear múltiples proveedores). Otra situación sería que la ley exija al cliente disponer de una estrategia adecuada previamente planificada que le permita garantizar la continuidad de las operaciones y evitar los efectos negativos de la cancelación o la suspensión de los servicios de computación en la nube para los usuarios finales. Algunas cláusulas contractuales pueden ayudar al cliente a reducir los riesgos de continuidad de las operaciones, especialmente en caso de insolvencia del proveedor y suspensión o cese unilateral de los servicios de computación en la nube.

Y cuando es el caso de que el cliente ya no quiera continuar con el proveedor, el cliente debería examinar con antelación el contenido sujeto a una posible estrategia de salida (por ejemplo, si será necesario retirar únicamente los datos que el cliente ha subido a la nube o también los datos obtenidos de los servicios de nube). El cliente podría necesitar también garantías de que tendrá acceso en el momento oportuno a las claves de descifrado custodia-

35 Los servicios de computación en la nube crecerán este año $40 \%$ en Latinoamérica, según previsiones del International Data Corporation (IDC), que estima en al menos US\$ 3600 millones el valor de las inversiones que las empresas realizarán en este modelo de tecnología por demanda. En particular, los servicios de nube benefician a los grupos de ventas y marketing, finanzas/contabilidad, producción/logística, así como al equipo de soporte y experiencia del cliente. Mientras que las áreas que se benefician del código abierto incluyen a web, portales, intercambio de datos y contenidos, prueba y desarrollo, servicios de nube y hosting, entre otras. Pero hay retos, entre los desafíos que plantea el código abierto, se citaron rubros como la sustentabilidad de los proyectos de open source en el largo plazo, la preocupación por el soporte provisto por el vendedor o la comunidad correspondiente y la carencia de recursos especializados, acceso el 19 de julio de 2018, https://sg.com.mx/buzz/am-rica-latina-un-terreno-f-rtil-para-los-servicios-nube-y-el-c-digo-abierto 
das por el proveedor o por terceros. Todos estos factores, además del tiempo necesario para exportar todos los datos y contenidos del cliente y hacer que estén plenamente disponibles en sus propios sistemas o en los de otro proveedor, deben tenerse en cuenta a la hora de negociar las cláusulas del contrato relativas a la financiación del servicio.

c) Otros aspectos precontractuales tales como revelación de información y confidencialidad. ${ }^{36}$ Finalmente entre otros aspectos de carácter precontractual se tiene la necesidad de revelarse información mutuamente proveedor y cliente, por cuanto la mayor claridad en la comunicación contribuirá a un auténtico consentimiento informado, so pena de que en caso de no cumplir este requisito, dicho contrato, o una parte de este, resulte nulo de pleno derecho o que la parte perjudicada pueda reclamar una indemnización por daños y perjuicios.

Adicionalmente, las partes en un futuro contrato tal vez necesiten llegar a un acuerdo sobre la confidencialidad de la información que se revelaría en la fase precontractual. Tal vez también necesiten firmar acuerdos de confidencialidad o contratos de no divulgación con los terceros que participen en el proceso de reunión de información antes de la celebración del contrato, en ejercicio de la diligencia debida (por ejemplo, los auditores).

De igual modo, antes de migrar datos a la nube, suele pedirse al cliente que clasifique los datos que va a migrar y los asegure en función de su grado de importancia y confidencialidad. Además de la transferencia de datos y contenidos desde los sistemas del cliente o desde su proveedor anterior a la nube del nuevo proveedor, la migración a la nube puede entrañar pruebas de instalación, configuración y cifrado y la formación del personal del cliente y otros usuarios finales. En estos casos, en ocasiones pueden surgir otros contratos independientes.

Como puede observarse, se trata de una etapa fundamental, que involucra que las partes tengan que ocuparse también de cuestiones relacionadas con los efectos de la revelación precontractual de información sobre la flexibilidad y la innovación en la etapa de ejecución del contrato, y seguramente muchos de estos acuerdos previos formarán parte del definitivo contrato en la nube.

Por otra parte, se recomienda que estos instrumentos aportados por la CNUDMI no proporcionen asesoramiento jurídico ni den la impresión de que favorecen a un tipo de parte en un contrato en vez de a otro. Debe recordarse que en el párrafo 15 de A/CN.9/902, en que figura el informe del Grupo de Trabajo IV (Comercio Electrónico), sobre la labor realizada en su $55^{\circ}$ período de sesiones, se insta a adoptar una posición neutral al señalarse que "tras deliberar, el Grupo de Trabajo decidió recomendar a la Comisión que se preparara una lista

36 Asamblea General de las Naciones Unidas, Comisión de las Naciones Unidas para el Derecho Mercantil Internacional, Grupo de Trabajo IV (Comercio Electrónico), 56 periodo de sesiones, Aspectos contractuales de la computación en la nube, Documento A/CN.9/WG.IV/WP.148, Nueva York, 16 a 20 de abril de 2018; 10-11. 
de las principales cuestiones que las partes contratantes podrían prever en los contratos de servicios de nube. En vista de la naturaleza de la lista, no se deberían ofrecer en ella recomendaciones ni orientación sobre las mejores prácticas". Postura que ha hecho que los Estados Unidos de América no apoyen la propuesta de la denominada Lista de verificación, por lo que continúa el trabajo a nivel de los organismos internacionales en afinar tales términos, siendo indispensable estos acuerdos, pues en este tipo de contratos existe una fuerte movilización transfronteriza de datos.

\section{CONCLUSIONES}

Existe una transformación digital que impulsa la innovación. Una de estas innovaciones la configura el contrato de prestación de servicios tecnológicos, denominado computación en la nube. Este modelo implica un cambio de paradigma que significa pagar por lo que se consume, teniendo la opción de multiplicidad de servicios en el ciberespacio o la singularidad de la prestación, siempre adecuándose a la necesidad del cliente; puede ser transfronterizo o circunscribirse a una jurisdicción, sea uno u otro el contexto o la característica bajo la cual se despliega este acuerdo técnico-jurídico, lo cierto es que es necesario reconocerlo en sus características esenciales a fin de regular sus aspectos más significativos.

No se discute que las tendencias actuales como la nube, la movilidad, la analítica / Big Data y el uso del código abierto han encontrado un terreno fértil, dando paso a nuevos servicios y aplicaciones más sofisticadas, lo que ha impactado positivamente en la rentabilidad de las organizaciones y del mismo ciberciudadano. Sin embargo, no debe olvidarse que también surgen riesgos frente a temas como la privacidad, la seguridad y la confianza, que gravitan alrededor de estos avances tecnológicos.

La existencia de este tipo de contrato que implica acceder de manera rápida y a bajo costo a centros de datos sin fronteras para la administración de aplicaciones, datos y dispositivos procura que organismos internacionales especializados armonicen a través de lineamientos los principales aspectos de su negociación, redacción y cumplimiento. En este sentido, una de las fases fundamentales de este tipo de acuerdo es la etapa preliminar y, por ello, se resalta lo importante de verificar la existencia de normas imperativas y otros requisitos cuando las partes acuerdan este tipo de modelo de negocios; la evaluación precontractual de riesgos y otros aspectos precontractuales tales como revelación de información y confidencialidad entre proveedores y clientes. 


\section{REFERENCIAS}

- Anónimo. "La computación en la nube cambia el paradigma de los negocios". Dinero (26 de mayo de 2016). Acceso el 17 de enero de 2019. https://www.dinero.com/edicionimpresa/tecnologia/articulo/la-computacion-en-la-nube-cambia-el-paradigma-de-losnegocios/224009

- Asamblea General de las Naciones Unidas. Comisión de las Naciones Unidas para el Derecho Mercantil Internacional. Grupo de Trabajo IV (Comercio Electrónico). $55^{\circ}$ periodo de sesiones. "Aspectos contractuales de la computación en la nube". Documento A/CN.9/ WG.IV/WP.142. Nueva York, 24 a 28 de abril de 2017.

- Asamblea General de las Naciones Unidas. Comisión de las Naciones Unidas para el Derecho Mercantil Internacional. Grupo de Trabajo IV (Comercio Electrónico). $56^{\circ}$ periodo de sesiones. "Aspectos contractuales de la computación en la nube". Documento A/CN.9/ WG.IV/WP.148. Nueva York, 16 a 20 de abril de 2018.

- Asamblea General de las Naciones Unidas. Comisión de las Naciones Unidas para el Derecho Mercantil Internacional. Grupo de Trabajo IV (Comercio Electrónico). $56^{\circ}$ periodo de sesiones. "Aspectos contractuales de los servicios de computación en la nube". Propuesta de los Estados Unidos de América. Documento A/CN.9/WG.IV/WP.151. Nueva York, 16 a 20 de abril de 2018.

- ENISA (Agencia Europea de Seguridad). "Seguridad y resistencia en las nubes de la Administración Pública”. ENISA. Acceso el 17 de enero de 2019. www.enisa.europa.eu/

- García del Poyo, Rafael. "Cloud computing: Aspectos jurídicos clave para la contratación de estos servicios". Revista Española de Relaciones Internacionales, n. 2 (2012). Acceso el 29 de abril de 2017. http://reri.difusionjuridica.es/index.php/RERI/article/view/45/43

- García Sánchez, M. "Retos de la computación en nube”. En Derecho y cloud computing, ed. por R. Martínez Martínez (Cizur Menor, Navarra: Thomson Reuters - Civitas, 2012).

- Zapata, Ricardo. "13,8 millones de venezolanos compraron o vieron una tienda online en 2017”. El Nacional Economía Empresa, 31 de mayo de 2018. Acceso el 21 de enero de 2019. http://www.el-nacional.com/noticias/empresas/138-millones-venezolanoscompraron-vieron-una-tienda-online-2017_237981

- Joyanes, Luis. "Computación en la nube. Notas para una estrategia española en cloud computing”. Revista del Instituto Español de Estudios Estratégicos, n. 0 (2012): 89-112.

- López Jiménez, David. "La "computación en la nube" o "cloud computing" examinada desde el ordenamiento jurídico espańol”. Revista de Derecho de la Pontificia Universidad 
Católica de Valparaíso, XL (2013, 1er Semestre): 689-709.

https://doi.org/10.4067/s0718-68512013000100021

- Marks, Eric A. y Bob Lozano. Executive's Guide to Cloud Computing. New Jersey: Wiley, 2010.

https://doi.org/10.1002/wics.139

- Smale, Will. "Cómo Drew Houston y Arash Ferdows triunfaron con Dropbox, una empresa tecnológica que Steve Jobs quiso destruir". BBC News. Economía (17 de julio de 2018). Acceso el 21 de enero de 2019. https://www.bbc.com/mundo/noticias-44847080

- Red de Gobierno Electrónico de América Latina y el Caribe (Red GEALC) Organización de los Estados Americanos (OEA), Banco Interamericano de Desarrollo (BID), Centro de Investigación para el Desarrollo Internacional (IDRC). "e-Gobierno en la nube". Boletín e-Gobierno Red GEALC 118 (octubre 2016).

RECIBIDO: 02/02/2019

APROBADO: 25/05/2019 